\title{
Indirect Ground-Water Discharge to the Great Lakes
}

U.S. GEOLOGICAL SURVEY

Open-File Report 98-579

Prepared in cooperation with

GREAT LAKES PROTECTION FUND

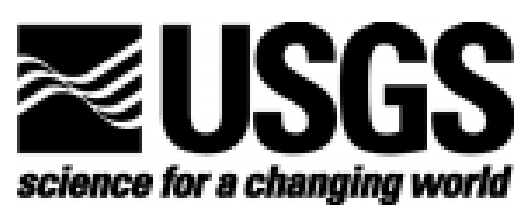




\section{Indirect Ground-Water Discharge to the Great Lakes}

By D.J. Holtschlag and J.R. Nicholas

Open-File Report 98-579

Lansing, Michigan

1998

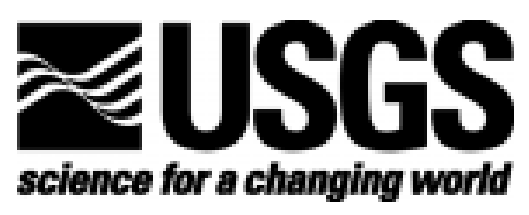




\section{U.S. DEPARTMENT OF THE INTERIOR BRUCE BABBITT, Secretary}

U.S. GEOLOGICAL SURVEY

Thomas J. Casadevall, Acting Director

For additional information write to:

District Chief

U.S. Geological Survey

6520 Mercantile Way, Suite 5

Lansing, Michigan 48911-5991
Copies of this report can be purchased from:

U.S. Geological Survey

Branch of Information Services

Box 25286

Denver, Colorado 80225-0286 


\section{CONTENTS}

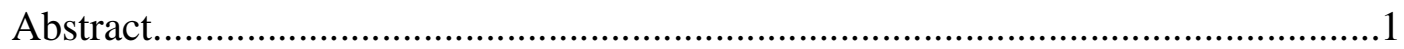

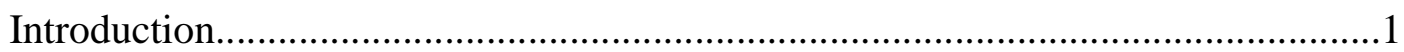

Indirect Ground-Water Discharge to the Great Lakes............................................2

Ground Water Component of Streamflow at Selected Gaging Stations...............2

Ground Water Component of Streamflow Discharging to the Great Lakes........5

Indirect Ground-Water Discharge as a Component of Basin Water Supply.........5

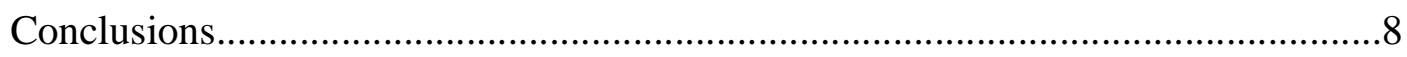

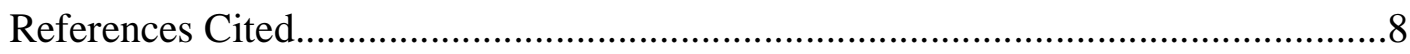

Appendix 1. Computation of indirect ground-water discharge to the Great Lakes

Basin

\section{FIGURES}

1-4. Maps showing-

1. Average ground-water component of streamflow for selected streams in the Great Lakes Basin.

2. Seasonal variability of the average ground-water and surfacerunoff components of streamflow for seven streams in the Great Lakes Basin.

3. Average ground-water and surface-runoff components of streamflow for the Great Lakes........................................................6

4. Basin water supply for the Great Lakes...........................................

\section{TABLE}

1. Ground-water component of streamflow for selected U.S. Geological Survey gaging stations in the Great Lakes Basin.......................................9

2. Basin water supply for the Great Lakes.....................................................5 
CONVERSION FACTORS

\begin{tabular}{rlll}
\hline Multiply & By & To obtain \\
\hline & Length & \\
inch (in.) & 25.4 & millimeter \\
& Area & \\
& & \\
square ${\text { mile }\left(\mathrm{mi}^{2}\right)}$ & 2.59 & square kilometer \\
& Flow & \\
cubic foot per second $\left(\mathrm{ft}^{3} / \mathrm{s}\right)$ & 0.02832 & cubic meter per second
\end{tabular}




\section{Indirect Ground-Water Discharge to the Great Lakes}

\author{
by D.J. Holtschlag and J.R. Nicholas
}

\section{ABSTRACT}

Estimates of the average ground-water component of streamflow for 195 streams in the United States part of the Great Lakes Basin range from 25 to 97 percent. Among the selected streams, the average ground-water component of streamflow was 67.3 percent. Estimates of the ground-water component of streamflow are based on hydrograph separation of 5,735 years of daily streamflow data. Incorporation of these estimates into the basin water supply for the Great Lakes shows that indirect discharge of ground water to the Great Lakes ranges from 22 percent of the basin water supply of Lake Erie to 42 percent of the basin water supply for Lake Ontario.

\section{INTRODUCTION}

Historically, scientists and water-resource managers have viewed ground water that flows directly from aquifers to the Great Lakes as a small fraction of the basin water supply. This perspective, however, does not account for the substantial amount of ground-water that flows indirectly into the Great Lakes as a component of streamflow.

In estimates of basin water supply for the Great Lakes, direct ground-water discharge generally is assumed to be small, typically within the uncertainty limits associated with estimates of precipitation, surface runoff, diversions, and evaporation. Recent work by Grannemann and Weaver (in press) summarizes studies of direct ground-water discharge to the Great Lakes and generally corroborates this assumption. They indicate that, while little is known about direct groundwater discharge to the Great Lakes, available information suggests that such direct discharge to the lakes is small. For Lake Michigan, the only Great Lake for which sufficient information is available to make a lake-wide estimate, direct ground-water discharge is only about 5 percent of the basin water supply for the lake. Direct ground-water discharge is difficult to estimate because it is determined by geologic and hydraulic properties that are difficult to measure locally and are highly variable from place to place.

Indirect ground-water discharge has not been explicitly considered in estimates of basin water supply for the Great Lakes. Instead, it has been incorporated into the streamflow component of basin water supply. Streamflow includes a surface-runoff component and a ground-water component. The surface-runoff component results from precipitation that flows overland to a stream or otherwise flows to a stream without percolating into an aquifer. The ground-water component results from that part of precipitation that infiltrates into the soil, percolates into an aquifer, and then flows to a stream. The ground-water component of streamflow in the Great Lakes Basin constitutes indirect ground-water discharge to the Great Lakes. Indirect ground-water discharge can be readily estimated from streamflow records that are available from gaging stations throughout the Great Lakes Basin.

In June 1998, the U.S. Geological Survey (USGS), in cooperation with the Great Lakes Protection Fund, began a study to summarize available data and information needs regarding the ground-water component of the Great Lakes basin water supply. This report presents estimates of indirect ground-water discharge made by applying hydrograph separation techniques to historical data from selected USGS streamgaging stations and relates these estimates to the Great Lakes basin water supply. 
The authors gratefully acknowledge the assistance of Nancy Baker, the Geographic Information Specialist for the USGS in Indiana, in the preparation of the illustrations. We also thank hydrologists at NOAA's Great Lakes Environmental Research Laboratory in Ann Arbor, Michigan, for assistance in retrieving and understanding basin water supply information for the Great Lakes.

\section{INDIRECT GROUND-WATER DIS- CHARGE TO THE GREAT LAKES}

The USGS has measured streamflow at 770 gaging stations in the Great Lakes Basin, some beginning as early as 1900 . About 300 of these stations currently are active. Data from USGS gaging stations provide a record of daily streamflow for flood forecasting, hydroelectric power generation, water-quality management, and regional streamflow information. In this report, an analysis of streamflow hydrographs is used to provide regional information on the indirect groundwater component of flow to the Great Lakes.

\section{Ground-Water Component of Stream- flow at Selected Gaging Stations}

Hydrograph separation provides a systematic method for identifying the groundwater and surface-runoff components of streamflow. The hydrograph separation method used in this report is based on a technique referred to as "streamflow partitioning" (Rutledge, 1993). This technique, implemented in the computer program PART (Rutledge, in press), scans the streamflow record for days that fit a requirement of antecedent recession (based on drainage area), designates ground-water flow to be equal to streamflow on these days, and then linearly interpolates the daily record of ground-water flow for days that do not fit the requirement of antecedent recession. The technique was selected because it was developed specifically to estimate the ground-water component of streamflow in the northeastern part of the United States, it produces estimates that are in close agreement with other manual and automated techniques, and it has data requirements that are consistent with available USGS data.

In this report, 5,735 years of daily streamflow values from 195 streamgaging stations, an average of 29.4 years per station, were analyzed to estimate indirect groundwater discharge to the Great Lakes. To provide appropriate estimates of indirect ground-water discharge, only those stations that satisfied the following criteria were selected: four or more years of continuous streamflow record were available, records were not significantly affected by streamflow regulation or diversion, and the stream drained an area smaller than 500 square miles.

Estimates of the average ground-water component of flow for the 195 streams range from 25 to 97 percent of the streamflow (fig. 1 and table 1). Among the selected gaging stations, the average ground-water component of streamflow was 67.3 percent. In general, the highest percentages of ground-water discharge are associated with basins having the highest percentages of land area covered by coarsetextured soils and undisturbed vegetation. Lowest percentages of ground-water discharge are associated with basins having the highest percentage of impervious areas or basins having the highest percentage of thin, tight soils overlying less permeable geologic materials. Like streamflow, the ground-water component of streamflow varies seasonally, generally reaching a maximum in March or April and a minimum in August or February (fig. 2). 


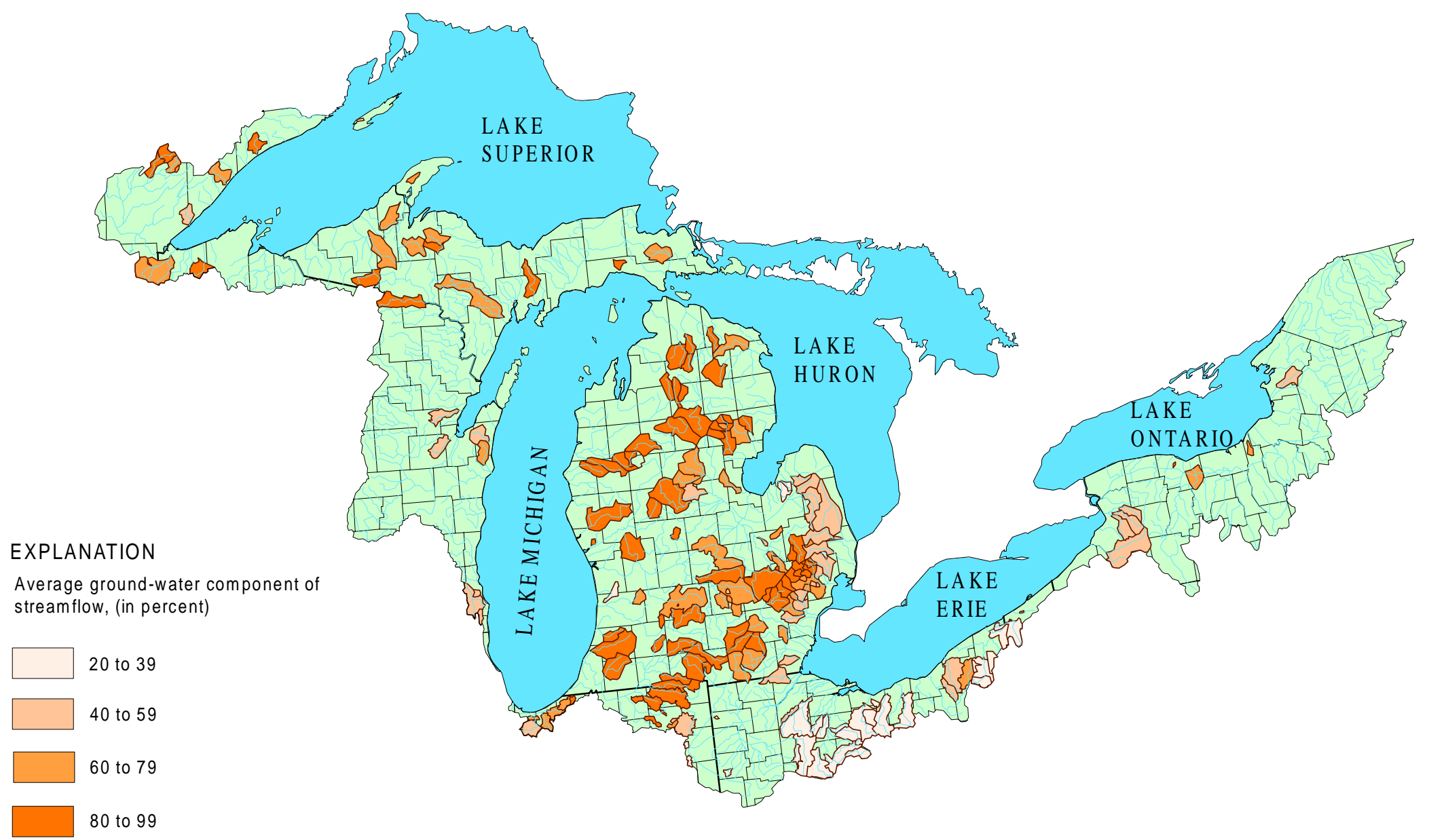

Figure 1. Average ground-water component of streamflow for selected streams in the Great Lakes. 

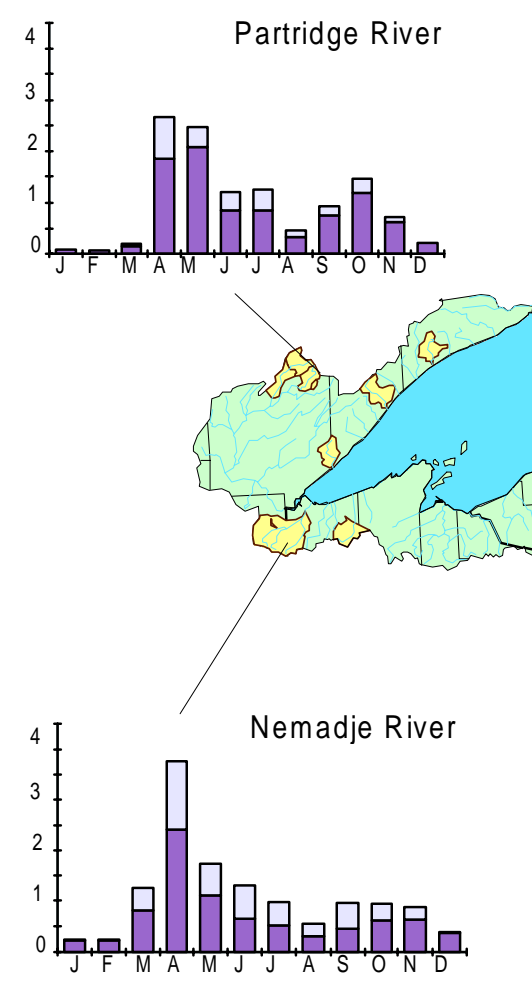

\section{EXPLANATION}

Average ground-water and surface-runoff components of streamflow, in inches

A surface runoff

Ground water

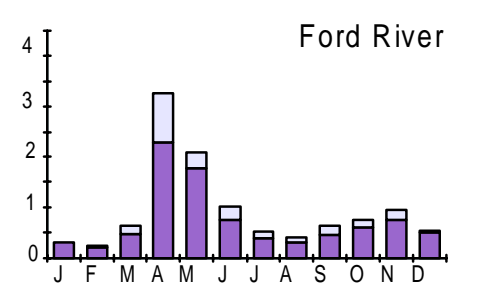

LAKE

SUPERIOR
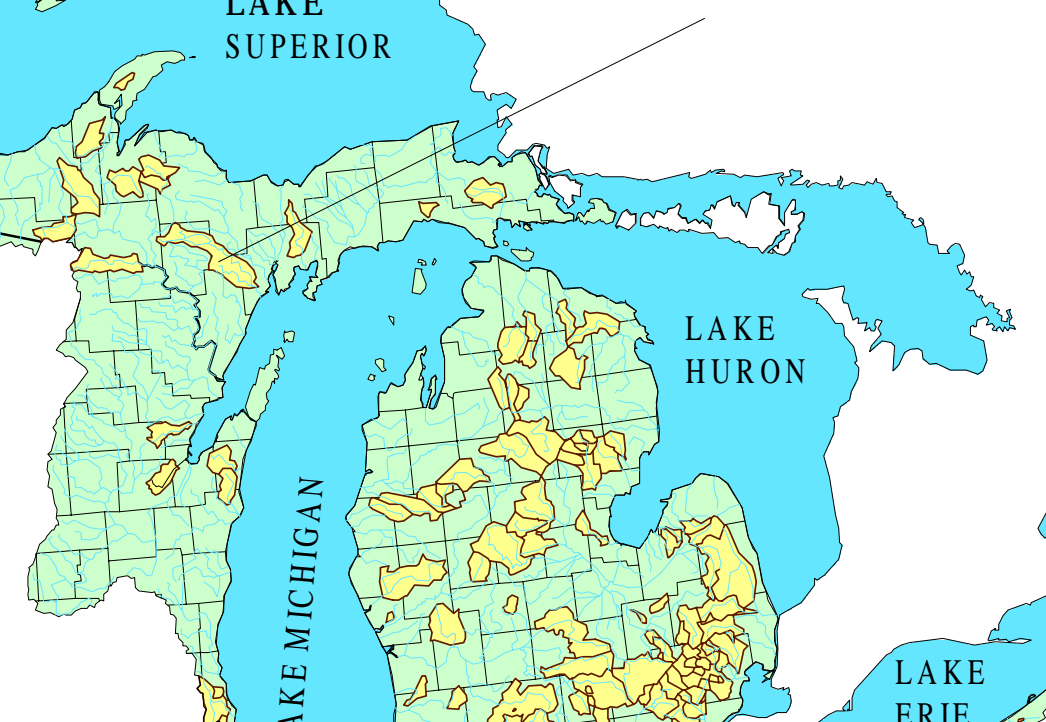

is
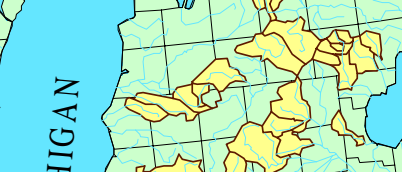

है के लिखो
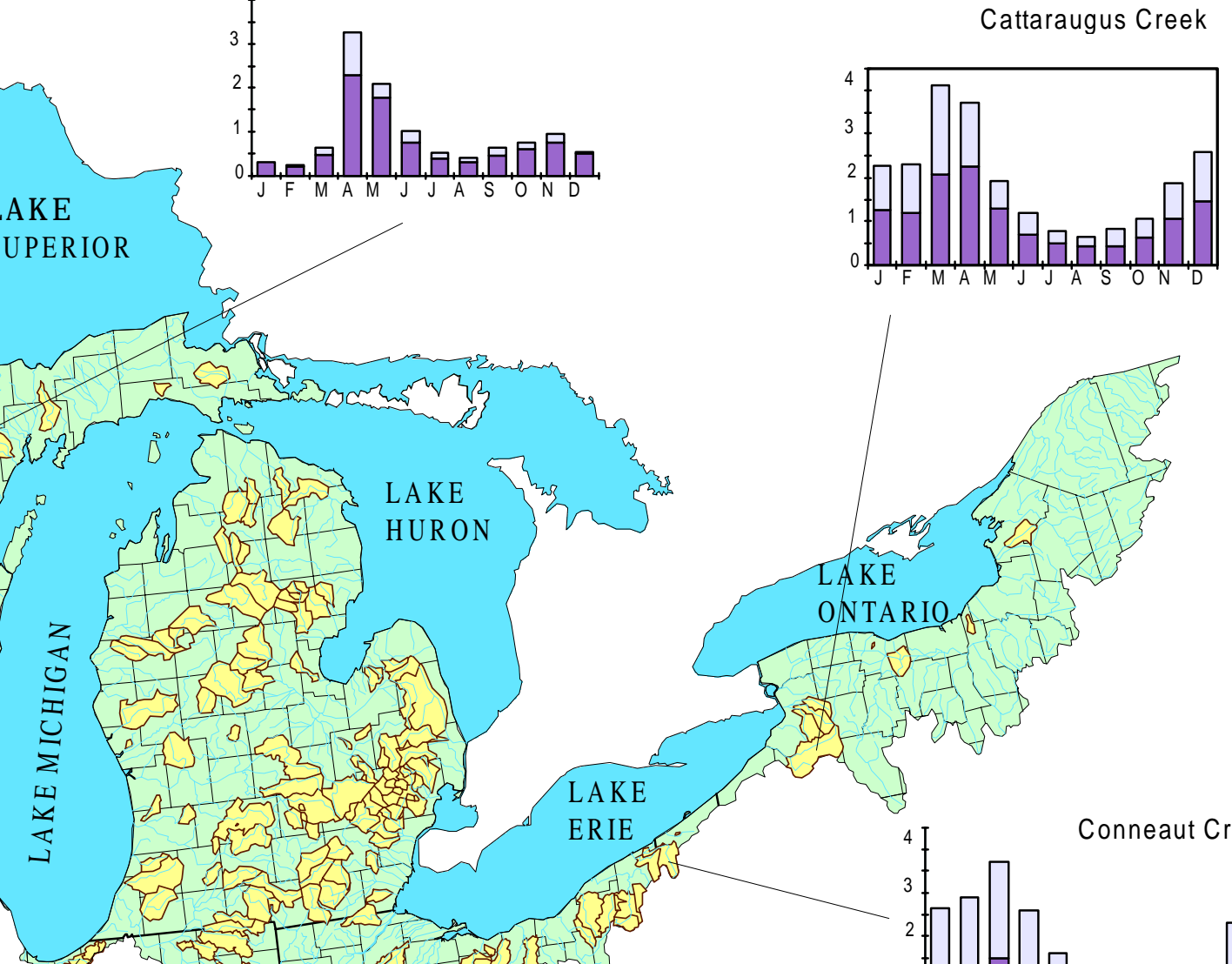

/ 
Table 2. Basin water supply for the Great Lakes.

\begin{tabular}{lccc}
\hline Lake & $\begin{array}{c}\text { Overlake } \\
\text { precipitation } \\
\text { (percent) }\end{array}$ & $\begin{array}{c}\text { Surface-runoff } \\
\text { (percent) }\end{array}$ & $\begin{array}{c}\text { Indirect ground- } \\
\text { water discharge } \\
\text { (percent) }\end{array}$ \\
\hline Superior & 56.3 & 11.0 & 32.7 \\
Michigan & 56.2 & 9.3 & 34.5 \\
Huron & 42.2 & 16.3 & 41.5 \\
Erie & 53.5 & 24.3 & 22.2 \\
Ontario & 34.8 & 22.8 & 42.4 \\
\hline
\end{tabular}

\section{Ground-Water Component of Stream- flow Discharging to the Great Lakes}

The drainage areas of the 195 gaging stations selected for hydrograph separation analysis represent only 13.6 percent of the total drainage area of the Great Lakes Basin. Thus, a large percentage of the Great Lakes Basin is not monitored by the selected gaging stations. These preliminary estimates of the total contribution of indirect ground-water to the basin water supply of the Great Lakes, therefore, could be improved by similar analysis of streamflow data available for Canadian streams and by quantitatively relating indirect ground-water discharge to selected physical characteristics of the entire Great Lakes Basin, such as was done by Holtschlag (1997) for Michigan's Lower Peninsula.

In this report, it is assumed that the average ground-water component of streamflow in a particular lake basin is equal to the average ground-water component of streamflow estimated from the gaged streams in that basin. Thus, the average ground-water component of streamflow for the Lake Superior, Lake Michigan, Lake Huron, Lake Erie, and Lake
Ontario Basins were computed on the basis of data from 15, 64, 56, 56, and 4 gaging stations, respectively. Using this approach, the average ground-water component of streamflow ranges from 48 percent for Lake Erie to 79 percent for Lake Michigan (fig. 3).

\section{Indirect Ground-Water Discharge as a Component of Basin Water Supply}

Croley and Hunter (1994) have compiled data on the total streamflow and overlake precipitation to describe the basin water supply for each Great Lake. The percentage groundwater component of streamflow for each Great Lake provides the basis for subdividing the total streamflow into indirect ground-water and surface-runoff components. This subdivision, generally based on data from 1950 to 1990, provides information on the ground-water component of the basin water supply that was not previously available. Results of these calculations (appendix 1) indicate that indirect ground-water discharge ranges from the smallest component of basin water supply for Lake Erie (22 percent) to the largest component of basin water supply for Lake Ontario (42 percent) (table 2 and fig. 4 ). 


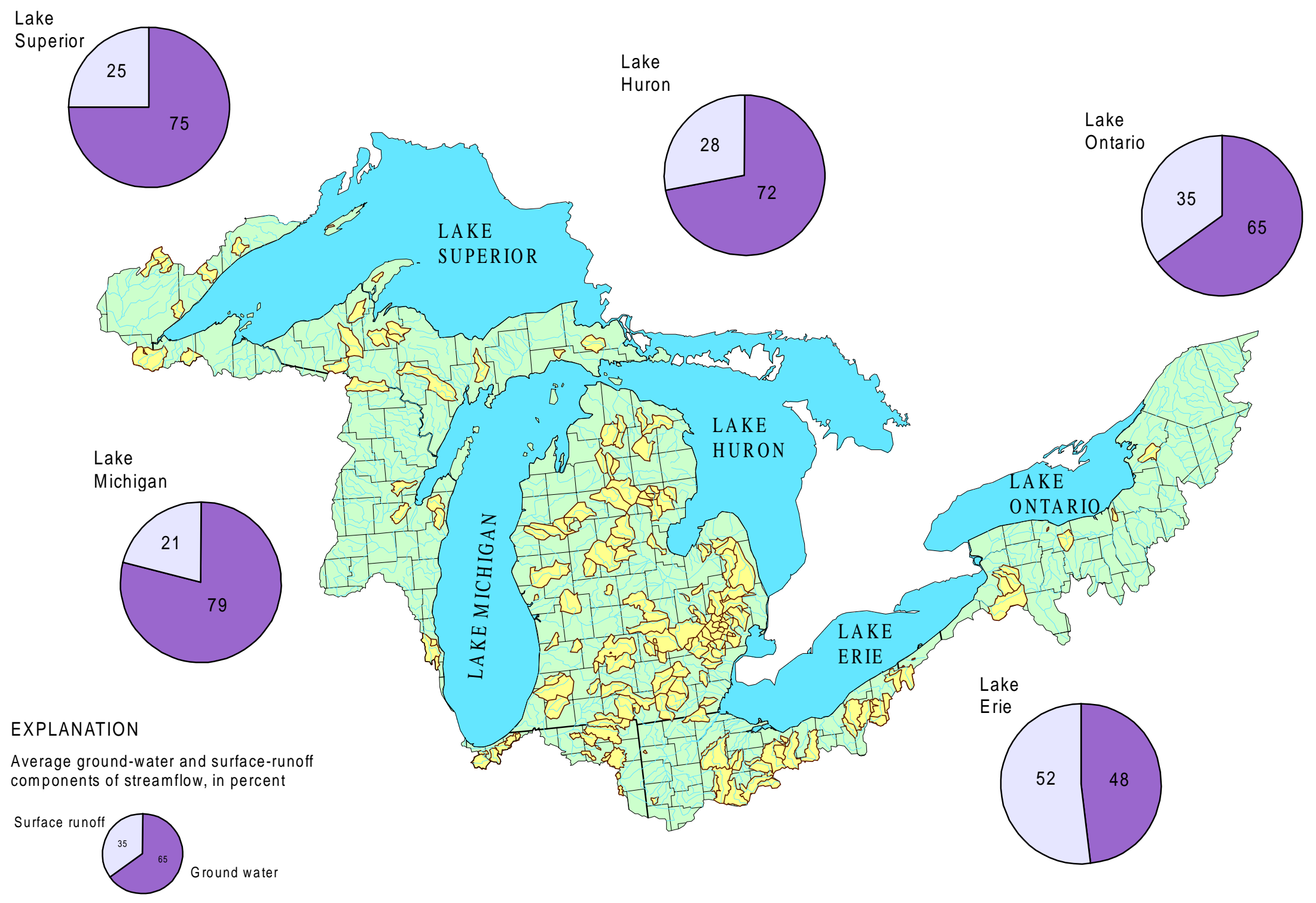

a

Figure 3. Average ground-water and surface-runoff components of streamflow for the Great Lakes. 


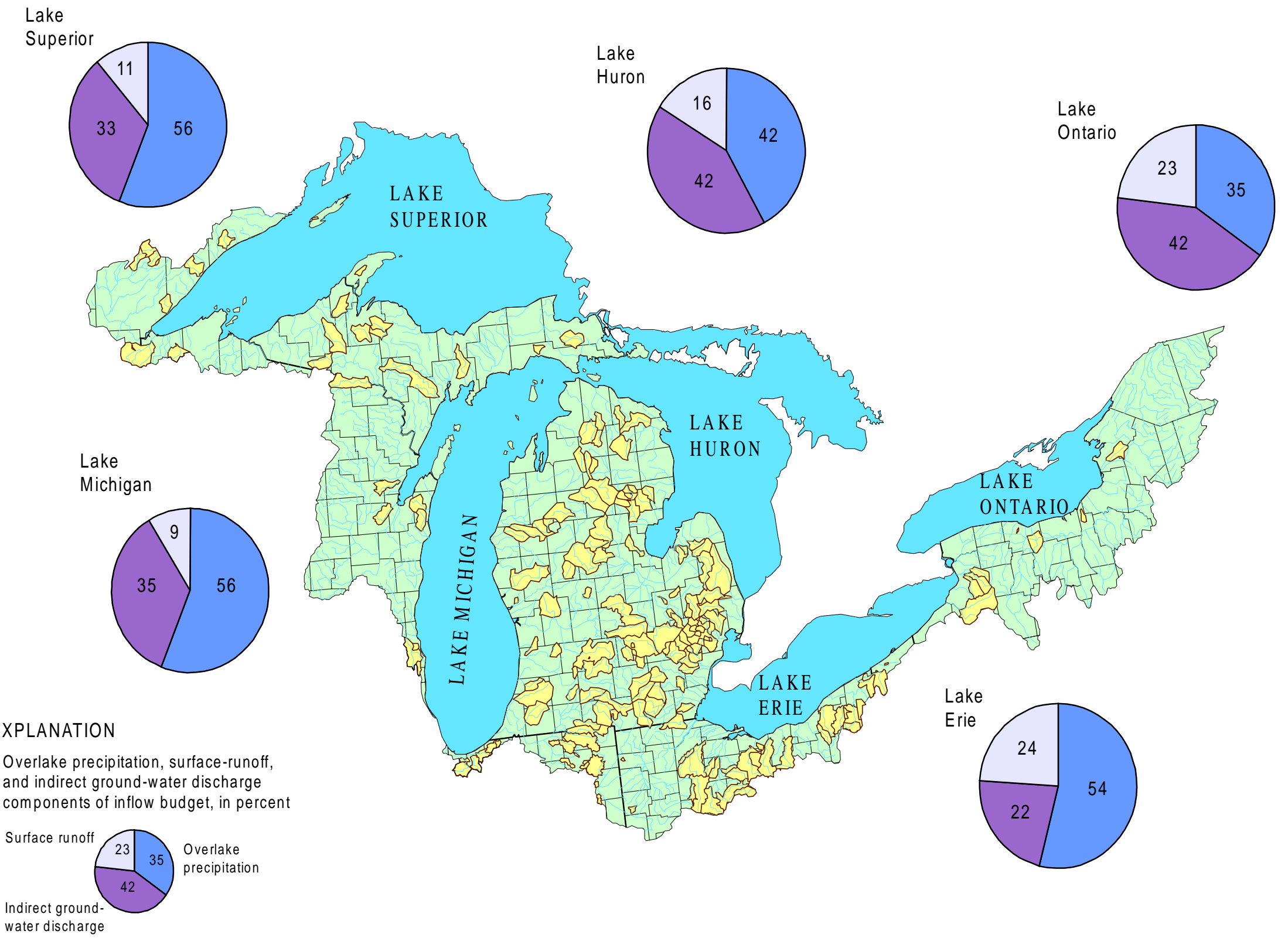

Figure 4. Basin water supply for the Great Lakes. 


\section{CONCLUSIONS}

Based on hydrograph separation analysis of streamflow data from 195 USGS gaging stations in the Great Lakes Basin, the average ground-water component of streamflow is 67.3 percent. This ground-water component of streamflow constitutes the indirect groundwater discharge to the Great Lakes, which ranges from 22 percent (Lake Erie) to 42 percent (Lake Ontario) of the basin water supply for the Great Lakes. Thus, even without explicitly accounting for direct ground-water discharge, ground water is a large component of the basin water supply of the Great Lakes.

\section{REFERENCES CITED}

Croley, T.E., II, and Hunter, T.S., 1994, Great Lakes monthly hydrologic data: National Oceanic and Atmospheric Administration Technical Report ERL GLERL-83, 84 p.

Grannemann, N.G. and Weaver, T.L., (in press), An annotated bibliography of selected references on the estimated rates of direct ground-water discharge to the Great Lakes: U.S. Geological Survey Water-Resources-Investigations Report 984039, 22 p.

Holtschlag, D.J., 1997, A generalized estimate of ground-water recharge rates in the Lower Peninsula of Michigan: U.S. Geological Survey Water-Supply Paper 2437, 37 p.

Rutledge, A.T., 1993, Computer programs for describing the recession of ground-water discharge and for estimating mean groundwater recharge and discharge from streamflow records: U.S. Geological Survey Water-Resources-Investigations Report 93-4121, 45 p.
Rutledge, A.T., (in press), Computer programs for describing the recession of groundwater discharge and for estimating mean ground-water recharge and discharge from streamflow records - update: U.S.

Geological Survey Water-ResourcesInvestigations Report 98-4148, $45 \mathrm{p}$. 
Table 1. Ground-water component of streamflow for selected U.S. Geological Survey gaging stations in the Great Lakes Basin

\begin{tabular}{|c|c|c|c|c|c|c|}
\hline $\begin{array}{c}\text { USGS } \\
\text { Station } \\
\text { number }\end{array}$ & Station name & $\begin{array}{c}\text { Drainage } \\
\text { area } \\
\text { (square } \\
\text { miles) }\end{array}$ & $\begin{array}{l}\text { Years } \\
\text { of } \\
\text { record }\end{array}$ & $\begin{array}{c}\text { Average } \\
\text { streamflow } \\
\text { (cubic feet } \\
\text { per second) }\end{array}$ & $\begin{array}{c}\text { Average } \\
\text { ground-water } \\
\text { component of } \\
\text { streamflow } \\
\text { (cubic feet per } \\
\text { second) }\end{array}$ & $\begin{array}{c}\text { Average } \\
\text { ground- } \\
\text { water } \\
\text { component } \\
\text { of } \\
\text { streamflow } \\
\text { (percent) }\end{array}$ \\
\hline \multicolumn{7}{|c|}{ STATIONS GAGING STREAMS TRIBUTARY TO LAKE SUPERIOR } \\
\hline 04001000 & Washington Creek at Windigo, MI & 13.2 & 33 & 16.4 & 12.4 & 75.6 \\
\hline 04012500 & Poplar River at Lutsen, MN & 112 & 21 & 106 & 89.5 & 84.8 \\
\hline 04014500 & Baptism River near Beaver Bay, MN & 140 & 59 & 169 & 110 & 65.3 \\
\hline 04015330 & Knife River near Two Harbors, MN & 85.6 & 22 & 93.0 & 48.9 & 52.6 \\
\hline 04015475 & Partridge River above Colby Lake at Hoyt Lakes, MN & 106 & 9 & 91.4 & 70.4 & 77.0 \\
\hline 04017000 & Embarrass River at Embarrass, MN & 88.3 & 22 & 64.5 & 51.9 & 80.5 \\
\hline 04018000 & Embarrass River near McKinley, MN & 171 & 8 & 109 & 93.1 & 85.6 \\
\hline 04024098 & Deer Creek near Holyoke, MN & 7.8 & 19 & 7.45 & 4.02 & 53.9 \\
\hline 04024430 & Nemadji River near South Superior, WI & 420 & 24 & 408 & 257 & 62.9 \\
\hline 04025500 & Bois Brule River at Brule, WI & 118 & 52 & 172 & 163 & 94.6 \\
\hline 04033000 & Middle Branch Ontonagon River near Paulding, MI & 164 & 52 & 170 & 152 & 89.5 \\
\hline 04035000 & East Branch Ontonagon River near Mass, MI & 272 & 36 & 257 & 194 & 75.6 \\
\hline 04040500 & Sturgeon River near Sidnaw, MI & 171 & 53 & 213 & 161 & 75.5 \\
\hline 04042500 & Otter River near Elo, MI & 162 & 29 & 215 & 161 & 75.1 \\
\hline 04043050 & Trap Rock River near Lake Linden, MI & 28 & 31 & 45.2 & 33.6 & 74.3 \\
\hline
\end{tabular}


Table 1. Ground-water component of streamflow for selected U.S. Geological Survey gaging stations in the Great Lakes Basin--continued

\begin{tabular}{|c|c|c|c|c|c|c|}
\hline $\begin{array}{c}\text { USGS } \\
\text { Station } \\
\text { number }\end{array}$ & Station name & $\begin{array}{l}\text { Drainage } \\
\text { area } \\
\text { (square } \\
\text { miles) }\end{array}$ & $\begin{array}{l}\text { Years } \\
\text { of } \\
\text { record }\end{array}$ & $\begin{array}{c}\text { Average } \\
\text { streamflow } \\
\text { (cubic feet } \\
\text { per second) }\end{array}$ & $\begin{array}{c}\text { Average } \\
\text { ground-water } \\
\text { component of } \\
\text { streamflow } \\
\text { (cubic feet per } \\
\text { second) }\end{array}$ & $\begin{array}{c}\text { Average } \\
\text { ground- } \\
\text { water } \\
\text { component } \\
\text { of } \\
\text { streamflow } \\
\text { (percent) }\end{array}$ \\
\hline
\end{tabular}

STATIONS GAGING STREAMS TRIBUTARY TO LAKE MICHIGAN

\begin{tabular}{|c|c|c|c|c|c|c|}
\hline 04046000 & Black River near Garnet, MI & 28 & 26 & 29.1 & 25.4 & 87.3 \\
\hline 04057510 & Sturgeon River near Nahma Junction, MI & 183 & 31 & 196 & 170 & 86.9 \\
\hline 04059500 & Ford River near Hyde, MI & 450 & 43 & 378 & 294 & 77.8 \\
\hline 04060993 & Brule River near Florence, MI & 366 & 52 & 353 & 312 & 88.3 \\
\hline 04062200 & Peshekee River near Champion, MI & 133 & 16 & 210 & 158 & 75.3 \\
\hline 04062230 & Michigamme River near Michigamme, MI & 194 & 13 & 283 & 252 & 89.1 \\
\hline 04071858 & Pensaukee River near Pensaukee, WI & 134 & 23 & 89.7 & 50.0 & 55.8 \\
\hline 04072150 & Duck Creek near Howard, WI & 108 & 9 & 56.6 & 25.0 & 44.1 \\
\hline 04085200 & Kewaunee River near Dewaunee, WI & 127 & 29 & 88.4 & 49.1 & 55.5 \\
\hline 04085281 & East Twin River at Mishicot, WI & 110 & 23 & 78.6 & 50.8 & 64.6 \\
\hline 04087204 & Oak Creek at South Milwaukee, WI & 25 & 34 & 23.1 & 9.92 & 43.0 \\
\hline 04087240 & Root River at Racine, WI & 190 & 34 & 151 & 79.7 & 52.8 \\
\hline 04087257 & Pike River at Racine, WI & 38.5 & 26 & 36.3 & 20.5 & 56.4 \\
\hline 04093000 & Deep River at Lake George Outlet, IN & 124 & 50 & 116 & 68.3 & 59.0 \\
\hline 04093500 & Burns Ditch at Gary, IN & 160 & 25 & 156 & 101 & 64.8 \\
\hline
\end{tabular}


Table 1. Ground-water component of streamflow for selected U.S. Geological Survey gaging stations in the Great Lakes Basin--continued

\begin{tabular}{|c|c|c|c|c|c|c|}
\hline $\begin{array}{c}\text { USGS } \\
\text { Station } \\
\text { number }\end{array}$ & Station name & $\begin{array}{c}\text { Drainage } \\
\text { area } \\
\text { (square } \\
\text { miles) }\end{array}$ & $\begin{array}{l}\text { Years } \\
\text { of } \\
\text { record }\end{array}$ & $\begin{array}{c}\text { Average } \\
\text { streamflow } \\
\text { (cubic feet } \\
\text { per second) }\end{array}$ & $\begin{array}{c}\text { Average } \\
\text { ground-water } \\
\text { component of } \\
\text { streamflow } \\
\text { (cubic feet per } \\
\text { second) }\end{array}$ & $\begin{array}{c}\text { Average } \\
\text { ground- } \\
\text { water } \\
\text { component } \\
\text { of } \\
\text { streamflow } \\
\text { (percent) }\end{array}$ \\
\hline 04094000 & Little Calumet River at Porter, IN & 66.2 & 50 & 78.0 & 54.0 & 69.2 \\
\hline 04094500 & Salt Creek at McCool, IN & 74.6 & 45 & 76.7 & 52.0 & 67.8 \\
\hline 04095300 & Trail Creek at Michigan City, IN & 54.1 & 25 & 76.4 & 56.5 & 73.9 \\
\hline 04096100 & Galena River near LaPorte, IN & 17.2 & 28 & 26.6 & 21.5 & 80.9 \\
\hline 04096405 & St. Joseph River at Burlington, MI & 206 & 35 & 178 & 161 & 90.7 \\
\hline 04096515 & South Branch Hog Creek near Allen, MI & 48.7 & 28 & 43.9 & 38.4 & 87.4 \\
\hline 04096600 & Coldwater Creek near Hodunk, MI & 293 & 26 & 254 & 226 & 88.8 \\
\hline 04096900 & Nottawa Creek near Athens, MI & 162 & 30 & 153 & 136 & 89.4 \\
\hline 04097170 & Portage River near Vicksburg, MI & 68.2 & 15 & 59.9 & 57.1 & 95.2 \\
\hline 04097540 & Prairie River near Nottawa, MI & 106 & 35 & 98.6 & 91.8 & 93.1 \\
\hline 04098500 & Fawn River near White Pigeon, MI & 192 & 17 & 157 & 145 & 92.6 \\
\hline 04099510 & Pigeon Creek near Angola, IN & 106 & 50 & 84.4 & 76.4 & 90.6 \\
\hline 04099610 & Pretty Lake near Stroh, IN & 2.0 & 17 & 0.48 & 0.39 & 80.8 \\
\hline 04099750 & Pigeon River near Scott, IN & 361 & 29 & 370 & 335 & 90.4 \\
\hline 04099808 & Lake Elkhart River at Middlebury, IN & 97.6 & 18 & 102 & 85.9 & 84.2 \\
\hline 04099850 & Pine Creek near Elkhart, IN & 31 & 18 & 19.8 & 16.5 & 83.1 \\
\hline 04100222 & North Branch Elkhart River at Cosperville, IN & 142 & 26 & 143 & 130 & 91.4 \\
\hline
\end{tabular}


Table 1. Ground-water component of streamflow for selected U.S. Geological Survey gaging stations in the Great Lakes Basin--continued

\begin{tabular}{|c|c|c|c|c|c|c|}
\hline $\begin{array}{c}\text { USGS } \\
\text { Station } \\
\text { number }\end{array}$ & Station name & $\begin{array}{c}\text { Drainage } \\
\text { area } \\
\text { (square } \\
\text { miles) }\end{array}$ & $\begin{array}{l}\text { Years } \\
\text { of } \\
\text { record }\end{array}$ & $\begin{array}{c}\text { Average } \\
\text { streamflow } \\
\text { (cubic feet } \\
\text { per second) }\end{array}$ & $\begin{array}{l}\text { Average } \\
\text { ground-water } \\
\text { component of } \\
\text { streamflow } \\
\text { (cubic feet per } \\
\text { second) }\end{array}$ & $\begin{array}{c}\text { Average } \\
\text { ground- } \\
\text { water } \\
\text { component } \\
\text { of } \\
\text { streamflow } \\
\text { (percent) }\end{array}$ \\
\hline 04100252 & Forker Creek near Burr Oak, IN & 19.2 & 28 & 17.9 & 14.2 & 79.6 \\
\hline 04100295 & Rimmell Branch near Albion, IN & 10.7 & 17 & 10.7 & 5.86 & 54.8 \\
\hline 04100377 & Solomon Creek near Syracuse, IN & 36.1 & 10 & 36.2 & 33.6 & 92.9 \\
\hline 04101800 & Dowagiac River at Sumnerville, MI & 255 & 37 & 301 & 270 & 89.6 \\
\hline 04102500 & Paw Paw River at Riverside, MI & 390 & 46 & 461 & 415 & 90.2 \\
\hline 04102700 & South Branch Black River near Bangor, MI & 83.6 & 31 & 107 & 86.2 & 80.7 \\
\hline 04105000 & Battle Creek at Battle Creek, MI & 241 & 64 & 210 & 175 & 83.7 \\
\hline 04105700 & Augusta Creek near Augusta, MI & 38.9 & 33 & 45.2 & 41.9 & 92.9 \\
\hline 04108600 & Rabbit River near Hopkins, MI & 71.4 & 32 & 61.6 & 47.5 & 77.2 \\
\hline 04108800 & Macatawa River near Zeeland, MI & 65.8 & 37 & 72.1 & 26.0 & 36.0 \\
\hline 04109000 & Grand River at Jackson, MI & 174 & 62 & 128 & 106 & 82.6 \\
\hline 04110000 & Orchard Creek at Munith, MI & 49 & 11 & 37.8 & 28.0 & 74.0 \\
\hline 04111500 & Deer Creek near Dansville, MI & 16.3 & 43 & 11.6 & 7.26 & 62.7 \\
\hline 04112000 & Sloan Creek near Williamston, MI & 9.3 & 43 & 5.91 & 3.20 & 54.1 \\
\hline 04112500 & Red Cedar River at East Lansing, MI & 355 & 65 & 215 & 154 & 71.6 \\
\hline 04112850 & Sycamore Creek near Holt, MI & 80.6 & 6 & 46.8 & 35.0 & 74.8 \\
\hline 04114500 & Looking Glass River near Eagle, MI & 281 & 51 & 186 & 159 & 85.4 \\
\hline
\end{tabular}


Table 1. Ground-water component of streamflow for selected U.S. Geological Survey gaging stations in the Great Lakes Basin--continued

\begin{tabular}{|c|c|c|c|c|c|c|}
\hline $\begin{array}{c}\text { USGS } \\
\text { Station } \\
\text { number }\end{array}$ & Station name & $\begin{array}{c}\text { Drainage } \\
\text { area } \\
\text { (square } \\
\text { miles) }\end{array}$ & $\begin{array}{l}\text { Years } \\
\text { of } \\
\text { record }\end{array}$ & $\begin{array}{c}\text { Average } \\
\text { streamflow } \\
\text { (cubic feet } \\
\text { per second) }\end{array}$ & $\begin{array}{c}\text { Average } \\
\text { ground-water } \\
\text { component of } \\
\text { streamflow } \\
\text { (cubic feet per } \\
\text { second) }\end{array}$ & $\begin{array}{c}\text { Average } \\
\text { ground- } \\
\text { water } \\
\text { component } \\
\text { of } \\
\text { streamflow } \\
\text { (percent) }\end{array}$ \\
\hline 04115000 & Maple River at Maple Rapids, MI & 434 & 53 & 283 & 224 & 78.9 \\
\hline 04115265 & Fish Creek near Crystal, MI & 39.7 & 10 & 35.0 & 29.4 & 84.2 \\
\hline 04117000 & Quaker Branch near Nashville, MI & 7.6 & 20 & 6.30 & 4.61 & 73.1 \\
\hline 04117500 & Thornapple River near Hastings, MI & 385 & 53 & 333 & 265 & 79.5 \\
\hline 04118500 & Rouge River near Rockford, MI & 281 & 39 & 243 & 205 & 84.4 \\
\hline 04121000 & Muskegon River near Merritt, MI & 355 & 27 & 230 & 212 & 92.1 \\
\hline 04121300 & Clam River at Vogel Center, MI & 243 & 31 & 136 & 121 & 89.0 \\
\hline 04121900 & Lake Muskegon River near Morley, MI & 138 & 29 & 133 & 116 & 87.5 \\
\hline 04122100 & Bear Creek near Muskegon, MI & 16.7 & 32 & 17.2 & 14.0 & 81.6 \\
\hline 04122200 & White River near Whitehall, MI & 406 & 40 & 455 & 413 & 90.7 \\
\hline 04123000 & Big Sable River near Freesoil, MI & 127 & 31 & 139 & 133 & 95.5 \\
\hline 04123500 & Manistee River near Grayling, MI & 159 & 31 & 184 & 179 & 97.0 \\
\hline 04125000 & Pine River near Le Roy, MI & 118 & 10 & 89.1 & 74.3 & 83.4 \\
\hline 04125460 & Pine River near Hoxeyville, MI & 245 & 29 & 288 & 263 & 91.2 \\
\hline 04126200 & Little Manistee River near Freesoil, MI & 200 & 18 & 174 & 164 & 94.5 \\
\hline \multicolumn{7}{|c|}{ STATIONS GAGING STREAMS TRIBUTARY TO LAKE HURON } \\
\hline 04127918 & Pine River near Rudyard, MI & 184 & 25 & 236 & 177 & 74.9 \\
\hline
\end{tabular}


Table 1. Ground-water component of streamflow for selected U.S. Geological Survey gaging stations in the Great Lakes Basin--continued

\begin{tabular}{|c|c|c|c|c|c|c|}
\hline $\begin{array}{c}\text { USGS } \\
\text { Station } \\
\text { number }\end{array}$ & Station name & $\begin{array}{l}\text { Drainage } \\
\text { area } \\
\text { (square } \\
\text { miles) }\end{array}$ & $\begin{array}{l}\text { Years } \\
\text { of } \\
\text { record }\end{array}$ & $\begin{array}{c}\text { Average } \\
\text { streamflow } \\
\text { (cubic feet } \\
\text { per second) }\end{array}$ & $\begin{array}{l}\text { Average } \\
\text { ground-water } \\
\text { component of } \\
\text { streamflow } \\
\text { (cubic feet per } \\
\text { second) }\end{array}$ & $\begin{array}{c}\text { Average } \\
\text { ground- } \\
\text { water } \\
\text { component } \\
\text { of } \\
\text { streamflow } \\
\text { (percent) }\end{array}$ \\
\hline 04127997 & Sturgeon River at Wolverine, MI & 192 & 54 & 219 & 200 & 91.3 \\
\hline 04128990 & Pigeon River near Vanderbilt, MI & 57.7 & 46 & 79.3 & 69.0 & 87.0 \\
\hline 04129500 & Pigeon River at Afton, MI & 159 & 38 & 140 & 122 & 87.4 \\
\hline 04131500 & Rainy River near Ocqueoc, MI & 87.9 & 27 & 42.5 & 34.7 & 81.6 \\
\hline 04132500 & Thunder Bay River near Hillman, MI & 232 & 27 & 215 & 193 & 90.0 \\
\hline 04134000 & North Branch Thunder Bay River near Bolton, MI & 184 & 34 & 118 & 92.2 & 78.0 \\
\hline 04135500 & Au Sable River at Grayling, MI & 110 & 50 & 76.4 & 72.0 & 94.3 \\
\hline 04135600 & East Branch Au Sable River at Grayling, MI & 76 & 25 & 44.2 & 42.0 & 95.2 \\
\hline 04135700 & South Branch Au Sable River near Luzerne, MI & 401 & 28 & 229 & 213 & 93.0 \\
\hline 04138000 & East Branch Au Gres River at McIvor, MI & 84 & 23 & 64.1 & 55.2 & 86.1 \\
\hline 04138500 & Au Gres River near National City, MI & 169 & 30 & 97.2 & 71.3 & 73.4 \\
\hline 04139000 & Houghton Creek near Lupton, MI & 29.7 & 22 & 51.2 & 45.5 & 88.9 \\
\hline 04139500 & Rifle River at the Ranch, MI & 56.8 & 20 & 91.5 & 82.6 & 90.2 \\
\hline 04140000 & Prior Creek near Selkirk, MI & 21.4 & 22 & 17.2 & 13.3 & 77.2 \\
\hline 04140500 & Rifle River at Selkirk, MI & 117 & 31 & 143 & 123 & 86.0 \\
\hline 04141000 & South Branch Shepards Creek near Selkirk, MI & 1.1 & 26 & 0.54 & 0.25 & 46.0 \\
\hline 04141500 & West Branch Rifle River near Selkirk, MI & 52 & 11 & 60.2 & 49.3 & 81.9 \\
\hline
\end{tabular}


Table 1. Ground-water component of streamflow for selected U.S. Geological Survey gaging stations in the Great Lakes Basin--continued

\begin{tabular}{|c|c|c|c|c|c|c|}
\hline $\begin{array}{c}\text { USGS } \\
\text { Station } \\
\text { number }\end{array}$ & Station name & $\begin{array}{c}\text { Drainage } \\
\text { area } \\
\text { (square } \\
\text { miles) }\end{array}$ & $\begin{array}{l}\text { Years } \\
\text { of } \\
\text { record }\end{array}$ & $\begin{array}{c}\text { Average } \\
\text { streamflow } \\
\text { (cubic feet } \\
\text { per second) }\end{array}$ & $\begin{array}{c}\text { Average } \\
\text { ground-water } \\
\text { component of } \\
\text { streamflow } \\
\text { (cubic feet per } \\
\text { second) }\end{array}$ & $\begin{array}{c}\text { Average } \\
\text { ground- } \\
\text { water } \\
\text { component } \\
\text { of } \\
\text { streamflow } \\
\text { (percent) }\end{array}$ \\
\hline 04142000 & Rifle River near Sterling, MI & 320 & 60 & 321 & 259 & 80.9 \\
\hline 04143500 & North Branch Kawkawlin River near Kawkawlin, MI & 101 & 30 & 59.0 & 38.8 & 65.8 \\
\hline 04144000 & Shiawassee River at Byron, MI & 368 & 35 & 251 & 202 & 80.3 \\
\hline 04146000 & Farmers Creek near Lapeer, MI & 55.3 & 64 & 32.6 & 26.2 & 80.4 \\
\hline 04146063 & South Branch Flint River near Columbiaville, MI & 221 & 17 & 185 & 150 & 81.0 \\
\hline 04147990 & Butternut Creek near Genesee, MI & 34.7 & 14 & 21.0 & 15.5 & 74.0 \\
\hline 04148200 & Swartz Creek near Holly, MI & 12.1 & 19 & 7.39 & 6.53 & 88.3 \\
\hline 04148300 & Swartz Creek at Flint, MI & 115 & 14 & 77.0 & 53.2 & 69.1 \\
\hline 04148440 & Thread Creek near Flint, MI & 54.4 & 14 & 35.6 & 26.9 & 75.6 \\
\hline 04148720 & Brent Run near Montrose, MI & 20.8 & 14 & 14.6 & 8.02 & 54.8 \\
\hline 04150000 & South Branch Cass River near Cass City, MI & 238 & 31 & 124 & 54.4 & 44.0 \\
\hline 04150500 & Cass River at Cass City, MI & 359 & 49 & 231 & 116 & 50.4 \\
\hline 04152238 & South Branch Tobacco River near Beaverton, MI & 160 & 11 & 133 & 104 & 78.6 \\
\hline 04152500 & Tobacco River at Beaverton, MI & 487 & 33 & 376 & 255 & 67.9 \\
\hline 04153500 & Salt River near North Bradley, MI & 138 & 36 & 78.0 & 39.1 & 50.2 \\
\hline 04154000 & Chippewa River near Mt Pleasant, MI & 416 & 65 & 328 & 278 & 84.8 \\
\hline 04157500 & State Drain near Sebewaing, MI & 67.3 & 14 & 34.7 & 11.0 & 31.6 \\
\hline
\end{tabular}


Table 1. Ground-water component of streamflow for selected U.S. Geological Survey gaging stations in the Great Lakes Basin--continued

\begin{tabular}{|c|c|c|c|c|c|c|}
\hline $\begin{array}{c}\text { USGS } \\
\text { Station } \\
\text { number }\end{array}$ & Station name & $\begin{array}{c}\text { Drainage } \\
\text { area } \\
\text { (square } \\
\text { miles) }\end{array}$ & $\begin{array}{l}\text { Years } \\
\text { of } \\
\text { record }\end{array}$ & $\begin{array}{c}\text { Average } \\
\text { streamflow } \\
\text { (cubic feet } \\
\text { per second) }\end{array}$ & $\begin{array}{l}\text { Average } \\
\text { ground-water } \\
\text { component of } \\
\text { streamflow } \\
\text { (cubic feet per } \\
\text { second) }\end{array}$ & $\begin{array}{c}\text { Average } \\
\text { ground- } \\
\text { water } \\
\text { component } \\
\text { of } \\
\text { streamflow } \\
\text { (percent) }\end{array}$ \\
\hline 04158000 & Columbia Drain near Sebewaing, MI & 33.9 & 14 & 17.2 & 4.54 & 26.5 \\
\hline 04158500 & Pigeon River near Owendale, MI & 53.2 & 29 & 32.4 & 18.7 & 57.9 \\
\hline 04159492 & Black River near Jeddo, MI & 464 & 52 & 306 & 126 & 41.3 \\
\hline 04159900 & Mill Creek near Avoca, MI & 169 & 20 & 95.7 & 54.4 & 56.8 \\
\hline 04160000 & Mill Creek near Abbottsford, MI & 208 & 16 & 102 & 58.9 & 57.9 \\
\hline 04160570 & North Branch Belle River at Imlay City, MI & 18.0 & 32 & 12.5 & 8.73 & 69.7 \\
\hline 04160600 & Belle River at Memphis, MI & 151 & 35 & 94.7 & 54.2 & 57.2 \\
\hline 04160800 & Sashabaw Creek near Drayton Plains, MI & 20.9 & 38 & 13.3 & 11.9 & 89.8 \\
\hline 04160900 & Clinton River near Drayton Plains, MI & 79.2 & 38 & 53.1 & 48.2 & 90.7 \\
\hline 04161100 & Galloway Creek near Auburn Heights, MI & 17.9 & 31 & 11.1 & 7.56 & 68.4 \\
\hline 04161500 & Paint Creek near Lake Orion, MI & 38.5 & 19 & 24.4 & 21.1 & 86.4 \\
\hline 04161540 & Paint Creek at Rochester, MI & 70.9 & 38 & 53.6 & 46.1 & 86.0 \\
\hline 04161580 & Stony Creek near Romeo, MI & 25.6 & 33 & 17.6 & 14.9 & 84.6 \\
\hline 04161800 & Stony Creek near Washington, MI & 68.2 & 39 & 43.6 & 37.0 & 84.9 \\
\hline 04163400 & Plum Branch at Utica, MI & 16.5 & 32 & 14.0 & 7.22 & 51.7 \\
\hline 04163500 & Plum Branch near Utica, MI & 22.9 & 12 & 11.6 & 5.95 & 51.2 \\
\hline 04164000 & Clinton River near Fraser, MI & 444 & 49 & 390 & 283 & 72.4 \\
\hline
\end{tabular}


Table 1. Ground-water component of streamflow for selected U.S. Geological Survey gaging stations in the Great Lakes Basin--continued

\begin{tabular}{|c|c|c|c|c|c|c|}
\hline $\begin{array}{c}\text { USGS } \\
\text { Station } \\
\text { number }\end{array}$ & Station name & $\begin{array}{c}\text { Drainage } \\
\text { area } \\
\text { (square } \\
\text { miles) }\end{array}$ & $\begin{array}{l}\text { Years } \\
\text { of } \\
\text { record }\end{array}$ & $\begin{array}{c}\text { Average } \\
\text { streamflow } \\
\text { (cubic feet } \\
\text { per second) }\end{array}$ & $\begin{array}{l}\text { Average } \\
\text { ground-water } \\
\text { component of } \\
\text { streamflow } \\
\text { (cubic feet per } \\
\text { second) }\end{array}$ & $\begin{array}{c}\text { Average } \\
\text { ground- } \\
\text { water } \\
\text { component } \\
\text { of } \\
\text { streamflow } \\
\text { (percent) }\end{array}$ \\
\hline 04164100 & East Pond Creek at Romeo, MI & 21.8 & 39 & 16.4 & 14.2 & 86.4 \\
\hline 04164300 & East Branch Coon Creek at Armada, MI & 13.0 & 39 & 7.45 & 2.72 & 36.5 \\
\hline 04164500 & North Branch Clinton River near Mount Clemens, MI & 199 & 49 & 129 & 64.3 & 49.9 \\
\hline 04164800 & Middle Branch Clinton River at Macomb, MI & 41.0 & 12 & 30.1 & 16.8 & 55.7 \\
\hline \multicolumn{7}{|c|}{ STATIONS GAGING STREAMS TRIBUTARY TO LAKE ERIE } \\
\hline 04166000 & River Rouge at Birmingham, MI & 33.3 & 47 & 20.8 & 14.1 & 67.9 \\
\hline 04166100 & River Rouge at Southfield, MI & 87.9 & 38 & 68.8 & 40.0 & 58.2 \\
\hline 04166200 & Evans Ditch at Southfield, MI & 9.5 & 39 & 8.91 & 3.56 & 39.9 \\
\hline 04166300 & Upper River Rouge at Farminton, MI & 17.5 & 39 & 13.8 & 8.66 & 62.9 \\
\hline 04166500 & River Rouge at Detroit, MI & 187 & 67 & 125 & 68.4 & 54.8 \\
\hline 04167000 & Middle River Rouge near Garden City, MI & 99.9 & 43 & 78.7 & 48.7 & 61.8 \\
\hline 04168000 & Lower River Rouge at Inkster, MI & 83.2 & 50 & 55.8 & 23.3 & 41.7 \\
\hline 04169500 & Huron River at Commerce, MI & 57.3 & 28 & 37.3 & 35.3 & 94.5 \\
\hline 04170000 & Huron River at Milford, MI & 132 & 49 & 102 & 89.8 & 88.3 \\
\hline 04171500 & Ore Creek near Brighton, MI & 31.0 & 16 & 21.2 & 19.4 & 91.7 \\
\hline 04172000 & Huron River near Hamburg, MI & 308 & 46 & 222 & 202 & 91.0 \\
\hline
\end{tabular}


Table 1. Ground-water component of streamflow for selected U.S. Geological Survey gaging stations in the Great Lakes Basin--continued

\begin{tabular}{|c|c|c|c|c|c|c|}
\hline $\begin{array}{c}\text { USGS } \\
\text { Station } \\
\text { number }\end{array}$ & Station name & $\begin{array}{l}\text { Drainage } \\
\text { area } \\
\text { (square } \\
\text { miles) }\end{array}$ & $\begin{array}{l}\text { Years } \\
\text { of } \\
\text { record }\end{array}$ & $\begin{array}{c}\text { Average } \\
\text { streamflow } \\
\text { (cubic feet } \\
\text { per second) }\end{array}$ & $\begin{array}{c}\text { Average } \\
\text { ground-water } \\
\text { component of } \\
\text { streamflow } \\
\text { (cubic feet per } \\
\text { second) }\end{array}$ & $\begin{array}{c}\text { Average } \\
\text { ground- } \\
\text { water } \\
\text { component } \\
\text { of } \\
\text { streamflow } \\
\text { (percent) }\end{array}$ \\
\hline 04175340 & Stony Creek at Oakville, MI & 68.0 & 11 & 44.4 & 28.5 & 64.2 \\
\hline 04175600 & River Raisin near Manchester, MI & 132 & 24 & 105 & 93.8 & 89.7 \\
\hline 04175700 & River Raisin near Tecumseh, MI & 267 & 23 & 180 & 149 & 82.9 \\
\hline 04176000 & River Raisin near Adrian, MI & 463 & 37 & 345 & 261 & 75.6 \\
\hline 04176605 & Otter Creek at La Salle, MI & 51.0 & 10 & 46.2 & 24.3 & 52.6 \\
\hline 04177000 & Ottawa River at Toledo, $\mathrm{OH}$ & 150 & 20 & 130 & 52.8 & 40.7 \\
\hline 04179500 & Cedar Creek near Auburn, IN & 87.3 & 29 & 68.4 & 41.4 & 60.5 \\
\hline 04180000 & Cedar Creek near Cedarville, IN & 270 & 50 & 256 & 147 & 57.6 \\
\hline 04182590 & Harbor Ditch at Fort Wayne, IN & 21.9 & 26 & 18.7 & 6.16 & 32.9 \\
\hline 04182810 & Spy Run at Fort Wayne, IN & 14.0 & 14 & 18.1 & 5.92 & 32.8 \\
\hline 04184500 & Bean Creek at Powers, $\mathrm{OH}$ & 206 & 39 & 168 & 109 & 65.2 \\
\hline 04185440 & Unnamed tributary to Lost Creek near Farmers, $\mathrm{OH}$ & 4.2 & 10 & 4.01 & 1.06 & 26.4 \\
\hline 04188500 & Eagle Creek near Findlay, OH & 55.0 & 9 & 43.7 & 11.2 & 25.7 \\
\hline 04189000 & Blanchard River near Findlay, OH & 346 & 50 & 270 & 87.9 & 32.5 \\
\hline 04191000 & Town Creek near Van Wert, OH & 21.2 & 6 & 23.8 & 5.88 & 24.7 \\
\hline 04195000 & North Branch Portage River near Bowling Green, $\mathrm{OH}$ & 45.1 & 7 & 42.5 & 21.3 & 50.0 \\
\hline 04195500 & Portage River at Woodville, $\mathrm{OH}$ & 428 & 11 & 334 & 84.1 & 25.2 \\
\hline
\end{tabular}


Table 1. Ground-water component of streamflow for selected U.S. Geological Survey gaging stations in the Great Lakes Basin--continued

\begin{tabular}{|c|c|c|c|c|c|c|}
\hline $\begin{array}{c}\text { USGS } \\
\text { Station } \\
\text { number }\end{array}$ & Station name & $\begin{array}{c}\text { Drainage } \\
\text { area } \\
\text { (square } \\
\text { miles) }\end{array}$ & $\begin{array}{l}\text { Years } \\
\text { of } \\
\text { record }\end{array}$ & $\begin{array}{c}\text { Average } \\
\text { streamflow } \\
\text { (cubic feet } \\
\text { per second) }\end{array}$ & $\begin{array}{l}\text { Average } \\
\text { ground-water } \\
\text { component of } \\
\text { streamflow } \\
\text { (cubic feet per } \\
\text { second) }\end{array}$ & $\begin{array}{c}\text { Average } \\
\text { ground- } \\
\text { water } \\
\text { component } \\
\text { of } \\
\text { streamflow } \\
\text { (percent) }\end{array}$ \\
\hline 04196000 & Sandusky River near Bucyrus, $\mathrm{OH}$ & 88.8 & 30 & 89.4 & 29.9 & 33.5 \\
\hline 04196500 & Sandusky River near Upper Sandusky, OH & 298 & 42 & 241 & 86.8 & 36.1 \\
\hline 04196800 & Tymochtee Creek at Crawford, $\mathrm{OH}$ & 229 & 6 & 170 & 51.6 & 30.4 \\
\hline 04197020 & Honey Creek near New Washington, $\mathrm{OH}$ & 17.0 & 8 & 16.1 & 6.26 & 39.0 \\
\hline 04197100 & Honey Creek at Melmore, $\mathrm{OH}$ & 149 & 20 & 132 & 43.0 & 32.6 \\
\hline 04197170 & Rock Creek at Tiffin, $\mathrm{OH}$ & 34.6 & 12 & 28.8 & 7.71 & 26.8 \\
\hline 04197300 & Wolf Creek at Bettsville, $\mathrm{OH}$ & 66.2 & 4 & 53.3 & 17.2 & 32.2 \\
\hline 04197450 & East Branch Wolf Creek near Bettsville, $\mathrm{OH}$ & 82.4 & 4 & 97.5 & 33.5 & 34.4 \\
\hline 04198500 & East Branch Huron River near Norwalk, OH & 85.5 & 10 & 65.3 & 22.4 & 34.3 \\
\hline 04199000 & Huron River at Milan, $\mathrm{OH}$ & 371 & 39 & 305 & 107 & 35.2 \\
\hline 04199155 & Old Womans Creek at Berlin Road near Huron, $\mathrm{OH}$ & 22.1 & 6 & 22.3 & 7.37 & 33.1 \\
\hline 04199500 & Vermilion River near Vermilion, $\mathrm{OH}$ & 262 & 30 & 258 & 84.1 & 32.6 \\
\hline 04200000 & East Branch Black River at Elyria, OH & 217 & 10 & 194 & 48.9 & 25.2 \\
\hline 04202000 & Cuyahoga River at Hiram Rapids, OH & 151 & 6 & 162 & 113 & 69.5 \\
\hline 04207200 & Tinkers Creek at Bedford, $\mathrm{OH}$ & 83.9 & 29 & 134 & 64.0 & 47.8 \\
\hline 04209000 & Chagrin River at Willoughby, $\mathrm{OH}$ & 246 & 6 & 410 & 173 & 42.3 \\
\hline 04209500 & Grand River near North Bristol, OH & 85.4 & 4 & 88.4 & 29.4 & 33.2 \\
\hline
\end{tabular}


Table 1. Ground-water component of streamflow for selected U.S. Geological Survey gaging stations in the Great Lakes Basin--continued

\begin{tabular}{|c|c|c|c|c|c|c|}
\hline $\begin{array}{c}\text { USGS } \\
\text { Station } \\
\text { number }\end{array}$ & Station name & $\begin{array}{c}\text { Drainage } \\
\text { area } \\
\text { (square } \\
\text { miles) }\end{array}$ & $\begin{array}{l}\text { Years } \\
\text { of } \\
\text { record }\end{array}$ & $\begin{array}{c}\text { Average } \\
\text { streamflow } \\
\text { (cubic feet } \\
\text { per second) }\end{array}$ & $\begin{array}{c}\text { Average } \\
\text { ground-water } \\
\text { component of } \\
\text { streamflow } \\
\text { (cubic feet per } \\
\text { second) }\end{array}$ & $\begin{array}{c}\text { Average } \\
\text { ground- } \\
\text { water } \\
\text { component } \\
\text { of } \\
\text { streamflow } \\
\text { (percent) }\end{array}$ \\
\hline 04210000 & Phelps Creek near Windsor, OH & 25.6 & 15 & 35.4 & 10.3 & 29.1 \\
\hline 04210500 & Grand River near Rome, $\mathrm{OH}$ & 251 & 4 & 274 & 109 & 39.9 \\
\hline 04211000 & Rock Creek near Rock Creek, OH & 69.2 & 23 & 75.4 & 21.3 & 28.2 \\
\hline 04212500 & Ashtabula River near Ashtabula, OH & 121 & 28 & 160 & 53.6 & 33.6 \\
\hline 04213000 & Conneaut Creek at Conneaut, $\mathrm{OH}$ & 175 & 46 & 290 & 109 & 37.7 \\
\hline 04213040 & Raccoon Creek near West Springfield, PA & 2.5 & 26 & 3.39 & 1.63 & 48.0 \\
\hline 04213075 & Brandy Run near Girard, PA & 4.4 & 8 & 7.16 & 3.65 & 51.0 \\
\hline 04213500 & Cattaraugus Creek at Gowanda, NY & 436 & 50 & 749 & 426 & 56.8 \\
\hline 04214500 & Buffalo Creek at Gardenville, NY & 142 & 50 & 208 & 93.2 & 44.9 \\
\hline 04215000 & Cayuga Creek near Lancaster, NY & 96.4 & 51 & 135 & 55.7 & 41.3 \\
\hline 04215500 & Cazenovia Creek at Ebenezer, NY & 135 & 50 & 237 & 104 & 43.9 \\
\hline \multicolumn{7}{|c|}{ STATIONS GAGING STREAMS TRIBUTARY TO LAKE ONTARIO } \\
\hline 04220262 & Northup Creek at North Greece, NY & 11.7 & 8 & 13.4 & 8.72 & 65.0 \\
\hline 04232050 & Irondequoit Creek, Rochester, NY & 142 & 7 & 139 & 93.5 & 67.2 \\
\hline 04232100 & Sterling Creek at Sterling, NY & 44.4 & 37 & 66.8 & 45.7 & 68.4 \\
\hline 04250750 & Sandy Creek at Adams, NY & 128 & 37 & 277 & 166 & 59.8 \\
\hline
\end{tabular}


Appendix 1. Computation of Indirect Ground-Water Discharge to the Great Lakes

\section{Lake Superior}

Basin Supply Item*

Runoff Lake.S := 24.16 in

Precip Lake.S := 31.14.in

Evap $_{\text {Lake.S }:=22.45 \cdot \text { in }}$

NBS Lake.S := $32.86 \cdot$ in

\section{Explanation}

Runoff is total streamflow from land areas within the basin.

Precip is overlake precipitation

Evap is evaporation from the lake surface.

NBS is net basin supply computed as Runoff + Precip - Evap.

*Based on monthly data from 1950 to 1990 as reported by Croley and Hunter (1994). All basin supply items are expressed as inches over the surface area of the corresponding lake. "S" denotes Superior.

\section{Physical Characteristics}

Area Lake.S $:=31700 \cdot \mathrm{mi}^{2} \quad$ Area of the lake surface.

Area Land.S $:=49300 \cdot \mathrm{mi}^{2} \quad$ Area of the land surface draining into the lake.

Area Basin.S:= Area Lake.S + Area Land.S

The average ground-water component of streamflow for runoff from the Lake

Superior basin (Groundwater.Percent.S) is based on hydrograph separation Groundwater Percent.S := 74.85.\% analysis of streamflow records from 15 USGS gaging stations.

\section{$\underline{\text { Results of Subdividing Runoff into Indirect-Ground Water and Surface-Runoff Components }}$}

Runoff Lake.IndirectGroundWater.S := Runoff ${ }_{\text {Lake.S }}$ Groundwater Percent.S

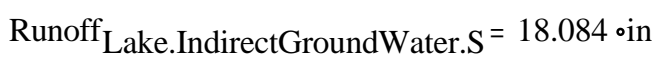

Runoff Lake.SurfaceRunoff.S : Runoff Lake.S· $(1$ - Groundwater Percent.S)

Runoff ${ }_{\text {Lake.SurfaceRunoff.S }}=6.076$ in

"Runoff.Lake" refers to the volume of runoff expressed in lake-area equivalent inches.

Annual runoff from land surface.

Runoff $_{\text {Land.IndirectGroundWater.S }:=\text { Runoff }}$ Lake.S ${ }^{\cdot A r e a}$ Lake.S $\cdot$ Groundwater $_{\text {Percent.S }}$ Area ${ }^{\text {Land.S }}{ }^{-1}$

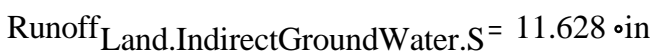

${ }_{\text {Runoff }}$ Land.SurfaceRunoffirectSurface. $S^{:}=$Runoff $_{\text {Lake.S }} \cdot{ }^{\cdot A r e a}$ Lake.S $\cdot\left(1-\right.$ Groundwater $\left._{\text {Percent.S }}\right) \cdot$ Area ${ }_{\text {Land.S }}{ }^{-1}$

Runoff $_{\text {Land.SurfaceRunoffirectSurface. }}=3.907$ in

"Runoff.Land" refers to the volume of runoff expressed in basin land-area equivalent inches. 
Appendix 1. Computation of Indirect Ground-Water Discharge to the Great Lakes--Continued

\section{Lake Michigan}

Basin Supply Item* Explanation

Runoff $_{\text {Lake.M }}:=25.11$ in Runoff is total streamflow from land areas within the basin.

Precip Lake.M $:=32.21 \cdot$ in Precip is overlake precipitation

Evap $_{\text {Lake.M }}:=25.18$ in Evap is evaporation from the lake surface.

NBS Lake.M $_{\text {: }} 32.14 \cdot$ in $\quad$ NBS is net basin supply computed as Runoff + Precip - Evap.

*Based on monthly data from 1950 to 1990 as reported by Croley and Hunter (1994). All basin supply items are expressed as inches over the surface area of the corresponding lake. "M" denotes Michigan.

\section{Physical Characteristics}

Area Lake.M := $22300 \cdot \mathrm{mi}^{2} \quad$ Area of the lake surface.

Area Land.M $:=45600 \cdot \mathrm{mi}^{2} \quad$ Area of the land surface draining into the lake.

Area Basin.M:= Area Lake.M + Area Land.M

The average ground-water component of streamflow for runoff from the Lake Michigan basin (Groundwater.Percent.M) is based on hydrograph Groundwater Percent.M := 78.68.\% separation analysis of streamflow records from 64 USGS gaging stations.

\section{Results of Subdividing Runoff into Indirect-Ground Water and Surface-Runoff Components}

Runoff ${ }_{\text {Lake.IndirectGroundWater.M := Runoff }}$ Lake.M Groundwater Percent.M

Runoff ${ }_{\text {Lake.IndirectGroundWater.M }}=19.757$ •in

Runoff $_{\text {Lake.SurfaceRunoffirectSurface.M:= Runoff }}$ Lake.M $\cdot\left(1\right.$ - Groundwater $\left.{ }_{\text {Percent.M }}\right)$

Runoff $_{\text {Lake.SurfaceRunoffirectSurface. }} M^{=} 5.353$ •in

"Runoff.Lake" refers to the volume of runoff expressed in lake-area equivalent inches.

Annual runoff from land surface.

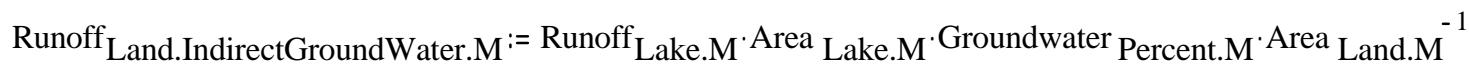

Runoff $_{\text {Land.IndirectGroundWater.M }}=9.662$ •in

Runoff $_{\text {Land.SurfaceRunoffirectSurface.M: }}{ }^{\text {Runoff }}{ }_{\text {Lake.M }}{ }^{\text {Area }}{ }_{\text {Lake.M}} \cdot\left(1\right.$ - Groundwater Percent.M $\cdot$ Area $^{\text {Land.M }}{ }^{-1}$

Runoff $_{\text {Land.SurfaceRunoffirectSurface. } M^{=}} 2.618$ in

"Runoff.Land" refers to the volume of runoff expressed in basin land-area equivalent inches. 
Appendix 1. Computation of Indirect Ground-Water Discharge to the Great Lakes--Continued

\section{Lake Huron}

Basin Supply Item ${ }^{*}$ Explanation

Runoff $_{\text {Lake.H }}:=45.78 \cdot$ in

Precip Lake.H $_{\text {:= } 33.43 \cdot \text { in }}$

Evap Lake.H $_{\text {L }}$ 24.20 in

$\mathrm{NBS}_{\text {Lake.H }}:=55.00 \cdot$ in
Runoff is total streamflow from land areas within the basin.

Precip is overlake precipitation

Evap is evaporation from the lake surface.

NBS is net basin supply computed as Runoff + Precip - Evap.

*Based on monthly data from 1955 to 1990 as reported by Croley and Hunter (1994). All basin supply items are expressed as inches over the surface area of the corresponding lake. "H" denotes Huron.

\section{Physical Characteristics}

Area Lake.H $:=23000 \cdot \mathrm{mi}^{2} \quad$ Area of the lake surface.

Area Land.H $:=51700 \cdot \mathrm{mi}^{2} \quad$ Area of the land surface draining into the lake.

Area Basin.H:= Area Lake.H + Area Land.H

The average ground-water component of streamflow for runoff from the Lake Huron basin (Groundwater.Percent.H) is based on hydrograph separation analysis of streamflow records from 56 USGS gaging stations.

\section{Results of Subdividing Runoff into Indirect-Ground Water and Surface-Runoff Components}

Runoff $_{\text {Lake.IndirectGroundWater.H }}$ := Runoff ${ }_{\text {Lake.H }}$ Groundwater Percent.H

Runoff $_{\text {Lake.IndirectGroundWater.H }}=32.879$ •in

Runoff $_{\text {Lake.SurfaceRunoffirectSurface.H }}:=$ Runoff $_{\text {Lake.H }} \cdot(1$ - Groundwater Percent.H $)$

Runoff ${ }_{\text {Lake.SurfaceRunoffirectSurface. } \mathrm{H}}=12.901$ •in

"Runoff.Lake" refers to the volume of runoff expressed in lake-area equivalent inches.

Annual runoff from land surface.

Runoff $_{\text {Land.IndirectGroundWater.H }}{ }^{=}$Runoff $_{\text {Lake.H }}{ }^{\cdot A r e a}$ Lake.H ${ }^{\text {Groundwater }}$ Percent.H ${ }^{\text {Area }}{ }^{\text {Land.H }}{ }^{-1}$

Runoff $_{\text {Land.IndirectGroundWater.H }}=14.627$ •in

Runoff $_{\text {Land.SurfaceRunoffirectSurface.H }}:=$ Runoff $_{\text {Lake.H }}{ }^{\text {Area }}$ Lake.H$\cdot(1-$ Groundwater Percent.H $) \cdot$ Area ${ }^{\text {Land.H }}{ }^{-1}$

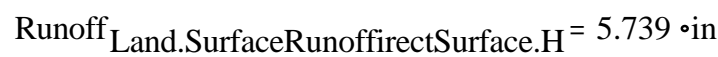

"Runoff.Land" refers to the volume of runoff expressed in basin land-area equivalent inches. 
Appendix 1. Computation of Indirect Ground-Water Discharge to the Great Lakes--Continued

\section{Lake Erie}

Basin Supply ltem* Explanation

Runoff ${ }_{\text {Lake.E }}:=31.63 \cdot$ in $\quad$ Runoff is total streamflow from land areas within the basin.

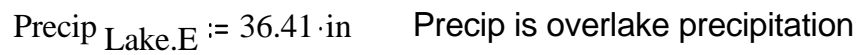

Evap $_{\text {Lake.E }}:=35.20$ in $\quad$ Evap is evaporation from the lake surface.

NBS Lake.E $:=32.84 \cdot$ in $\quad$ NBS is net basin supply computed as Runoff + Precip - Evap.

*Based on monthly data from 1950 to 1990 as reported by Croley and Hunter (1994). All basin supply items are expressed as inches over the surface area of the corresponding lake. "E" denotes Erie.

Physical Characteristics

Area Lake.E $:=9910 \cdot \mathrm{mi}^{2} \quad$ Area of the lake surface.

Area Land.E $:=30140 \cdot \mathrm{m}^{2} \quad$ Area of the land surface draining into the lake.

Area Basin.E:= Area Lake.E + Area Land.E

The average ground-water component of streamflow for runoff from the Lake

Erie basin (Groundwater.Percent.E) is based on hydrograph separation $\quad$ Groundwater Percent.E $:=47.82 . \%$ analysis of streamflow records from 56 USGS gaging stations.

$\underline{\text { Results of Subdividing Runoff into Indirect-Ground Water and Surface-Runoff Components }}$

Runoff $_{\text {Lake.IndirectGroundWater.E: }}$ Runoff $_{\text {Lake.E }}$ Groundwater $^{\text {Percent.E }}$

Runoff ${ }_{\text {Lake.IndirectGroundWater.E }}=15.125$ in

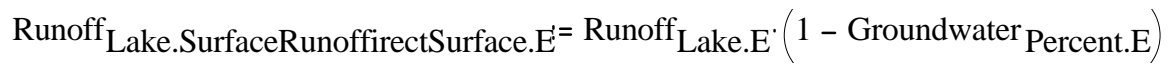

Runoff $_{\text {Lake.SurfaceRunoffirectSurface. }} \mathrm{E}^{=16.505 \text { in }}$

"Runoff.Lake" refers to the volume of runoff expressed in lake-area equivalent inches.

Annual runoff from land surface.

Runoff $_{\text {Land.IndirectGroundWater.E }}{ }^{\text {Runoff }}$ Lake.E $^{\text {Area }}{ }_{\text {Lake.E }}{ }^{\text {Groundwater }}{ }_{\text {Percent.E }}{ }^{\text {Area }}{ }^{\text {Land.E }}{ }^{-1}$

Runoff ${ }_{\text {Land.IndirectGroundWater. }}=4.973$ oin

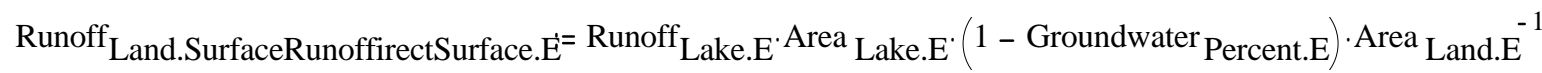

Runoff Land.SurfaceRunoffirectSurface.E 5.427 in

"Runoff.Land" refers to the volume of runoff expressed in basin land-area equivalent inches. 
Appendix 1. Computation of Indirect Ground-Water Discharge to the Great Lakes--Continued Lake Ontario

Basin Supply Item* Explanation

Runoff ${ }_{\text {Lake.O }}:=64.08$.in Runoff is total streamflow from land areas within the basin.

Precip $_{\text {Lake.O }}:=34.14 \cdot$ in $\quad$ Precip is overlake precipitation

Evap $_{\text {Lake.O }}:=25.40 \cdot$ in $\quad$ Evap is evaporation from the lake surface.

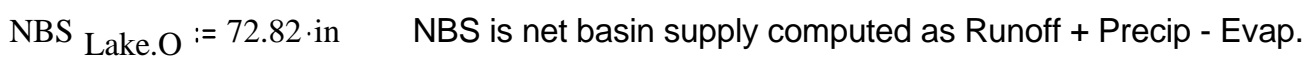

*Based on monthly data from 1950 to 1990 as reported by Croley and Hunter (1994). All basin supply items are expressed as inches over the surface area of the corresponding lake. "O" denotes Ontario.

\section{Physical Characteristics}

Area Lake.O := $7340 \cdot \mathrm{mi}^{2} \quad$ Area of the lake surface.

Area Land.O $:=24720 \cdot \mathrm{mi}^{2} \quad$ Area of the land surface draining into the lake.

Area Basin.O := Area Lake.O + Area Land.O

The average ground-water component of streamflow for runoff from the Lake Ontario basin (Groundwater.Percent.O) is based on hydrograph separation analysis of streamflow records from 4 USGS gaging stations.

\section{Results of Subdividing Runoff into Indirect-Ground Water and Surface-Runoff Components}

Runoff Lake.IndirectGroundWater.O $:=$ Runoff $_{\text {Lake.O }}$ Groundwater Percent.O

Runoff ${ }_{\text {Lake.IndirectGroundWater.O }}=41.716$ in

Runoff ${ }_{\text {Lake.SurfaceRunoffirectSurface.O }}$ : Runoff $_{\text {Lake.O }}$ ( 1 - Groundwater Percent.O $)$

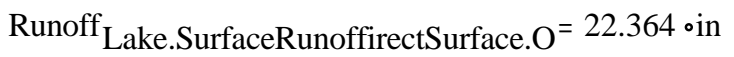

"Runoff.Lake" refers to the volume of runoff expressed in lake-area equivalent inches.

Annual runoff from land surface.

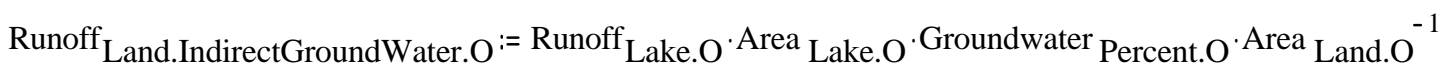

Runoff Land.IndirectGroundWater. $=12.387$ in

Runoff Land.SurfaceRunoffirectSurface.O:= Runoff ${ }_{\text {Lake.O }}{ }^{\text {Area }}{ }^{\text {Lake.O }} \cdot(1$ - Groundwater Percent.O $) \cdot$ Area ${ }^{\text {Land.O }}{ }^{-1}$

Runoff $_{\text {Land.SurfaceRunoffirectSurface. }} \mathrm{O}^{=} 6.64$ •in

"Runoff.Land" refers to the volume of runoff expressed in basin land-area equivalent inches. 